\title{
The importance of visualisation of results from slope stability
} analysis

\author{
J.-P. Renaud, M. G. Anderson, P. L. Wilkinson, D. M. Lloyd and D. Muir Wood
}

\section{R. Baker, Israel Institute of Technology; and D. Leshchinksy, University of Delaware \\ Employing a slightly different perspective, the authors utilised the notion of safety maps introduced by Baker and Leshchinsky ${ }^{9}$; in order to discuss results based on Bishop's simplified method of slices. In general their results are consistent with the more general framework introduced by Baker and Leshchinsky. However the following comments might be appropriate with respect to this work.}

1: The authors write 'Baker'; introduced the concept of a safety map, which consists in plotting, within the slope itself, the factor of safety associated with each slip surface: i.e. ... For the record, reference (9) was written by Baker and Leshchinsky, not Baker alone. More importantly, the above statement is essentially meaningless; infinite number of slip circles pass through each point in the slope. Each circle is associated with a different safety factor; and it is impossible to plot safety factors associated with each one of these circles at the same spatial point. The sentence following the 'i.e.' correctly characterises the safety map notion.

2: They write 'The first outcome of this visualisation method is that the critical slip surface expected by standard approach to this problem will naturally appear as a single circular arc (as shown in Fig. 5)'. This sentence is true but trivial; it is equivalent to the statement 'the critical circle is a circle'. The significant statement related to conventional critical slip surfaces is the first general relationship proved by Baker and Leshchinsky; namely that the conventional critical slip surface is identical to the minimal contour line in the safety map. This result is valid for all types of slip surfaces, not only circles.

3: Figure 4 is nothing more then an 'experimental' verification of the second general relation (the tangency theorem), which was proved by Baker and Leshchinsky. Again, this general result is valid for arbitrarily shaped slip surfaces, not only circles.

4: The critical slip surface (circle) emerging above the toe of the slope (Fig. 5(b) is obviously wrong. In the limiting equilibrium framework such a surface defines a slope having a 'reduced height'. For the homogeneous soil conditions considered by the authors, the critical slip surface must emerge either at the toe (toe failure), or below the toe (base failure), but never on the inclined face of the slope.

\begin{abstract}
Authors' reply
We are grateful to Baker and Leshchinsky for their interest in our paper which had the aim of investigating which visualisation techniques are best suited for practising engineers who very often use the Bishop simplified limit-equilibrium analysis together with a slip search algorithm. Our main conclusion was that it is important to retain as much as possible of the information gathered during the search for the critical slip circle, especially the safety factor values, and that a safety map technique such as that introduced by Baker and Leshchinsky seemed the most suitable technique to visualise the results.
\end{abstract}

Baker and Leshchinsky ${ }^{9}$; introduced the concept of a safety map which consists of plotting, for each point within the slope itself, the minimum factor of safety of all the slip surfaces passing though this point. Theoretically there is an infinite number of slip surfaces passing through each point in the slope and Baker and Leshchinsky prove elegantly that each slip surface is tangent to the contour line with the same factor of safety.

In any practical application using the Bishop simplified method, the number of slip surfaces studied becomes a finite number of circles. The fact that the critical slip surface corresponds to the lowest contour line of the safety map is not trivial in the sense that practising engineers will, by using a safety map approach to their visualisation, not only retain the common output from their slope stability package (namely the critical slip circle) but also gather more spatial information on the distribution of factors of safety. This information is then available to assist the engineer in a more complete interpretation of potentially weak zones within the slope. Fig. 4 is a graphical explanation of the process that we followed to produce safety maps associated with a conventional Bishop slip search algorithm. It illustrates what a contour line actually represents in the final safety map and we have found that a full exposition of the graphical linkages is important and of value to potential users of this procedure.

The slope stability calculations have been repeated following the discovery of an error in the computer code in the treatment of the circles that intersect the slope surface more than twice. For the $30^{\circ}$ slope the results were unchanged. Revised versions of Fig 5(b) and Fig 2(b) are included here. The revised version of Fig 5(b) also includes the grid centre contour plot (to be 


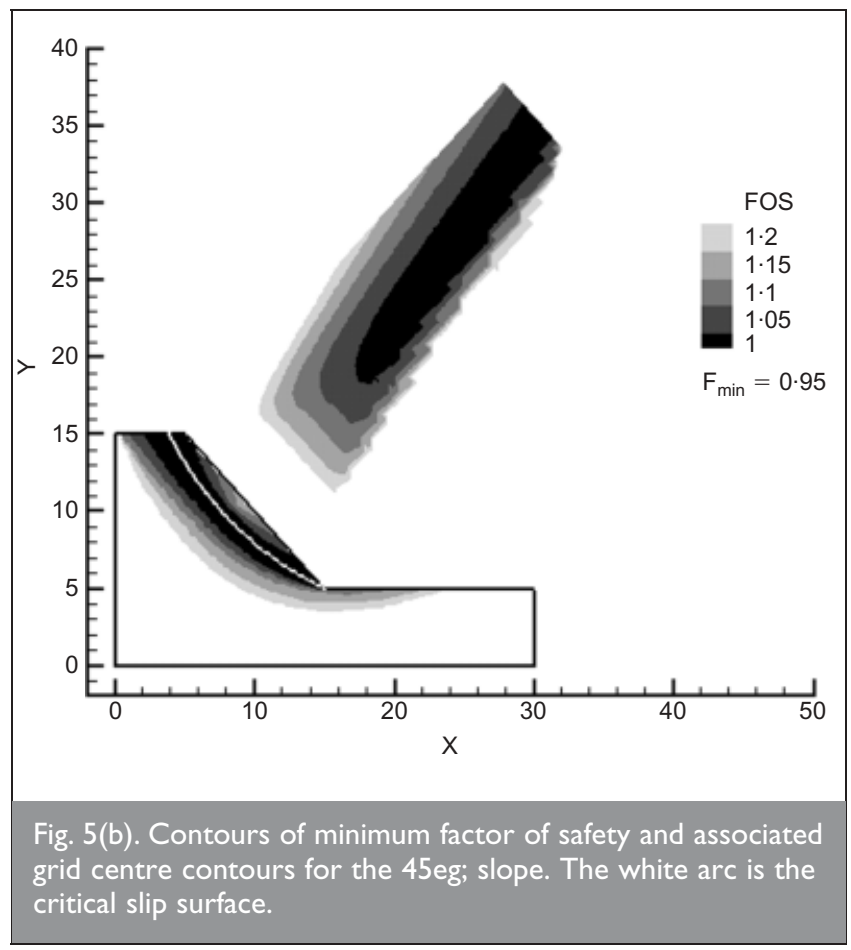

compared with Fig. 3(b) in the original paper). It is concluded that the fundamental benefits remain unchanged: the safety map is the only technique that gives clear visual indication of the spatial variability shown in the scatter plot.

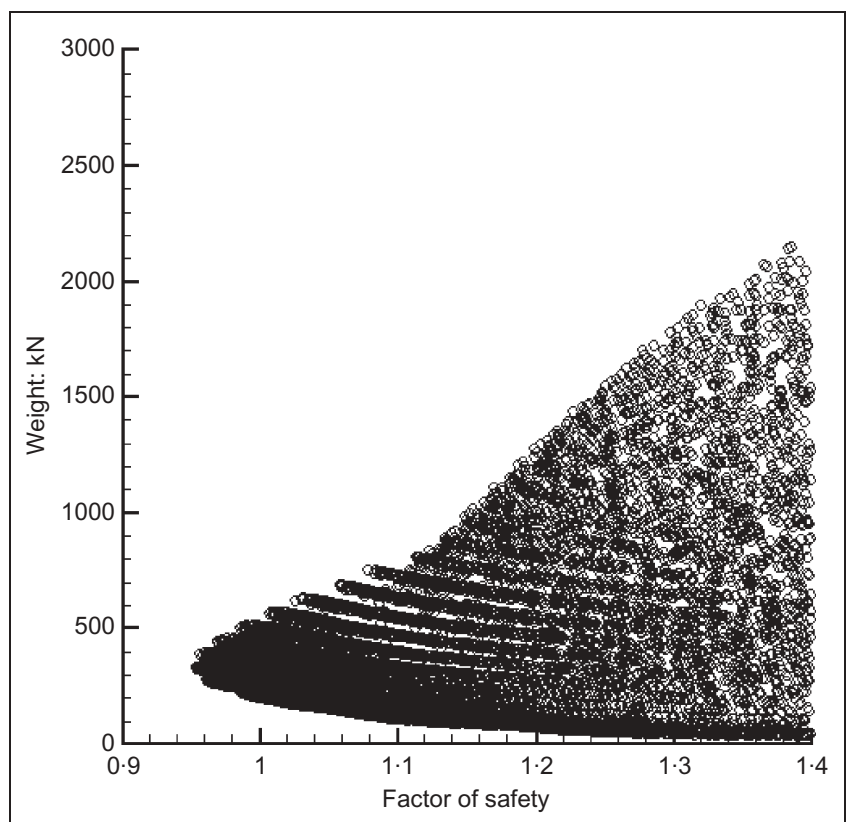

Fig. 2(b). Scatter plot of weight and factor of safety for each slip surface of the 45 degree slope.

\section{REFERENCES}

9. R. BAKER and D. LESHCHINSKY. Spatial distribution of safety factors. Journal of Geoenvironmental and Geotechnical Engineering, 2001, 127, No. 2, 135-145. 